\title{
Underwater SLAM in a Marina Environment
}

\author{
David Ribas, Pere Ridao, Juan Domingo Tardós and José Neira
}

\begin{abstract}
This paper describes a navigation system for autonomous underwater vehicles (AUVs) in partially structured environments, such as dams, harbors, marinas or marine platforms. A mechanical scanning imaging sonar is used to obtain information about the location of planar structures present in such environments. A modified version of the Hough transform has been developed to extract line features, together with their uncertainty, from the continuous sonar dataflow. The information obtained is incorporated into a feature-based SLAM algorithm running an Extended Kalman Filter (EKF). Simultaneously, the AUV's position estimate is provided to the feature extraction algorithm to correct the distortions that the vehicle motion produces in the acoustic images. Experiments carried out in a marina located in the Costa Brava (Spain) with the Ictineu AUV show the viability of the proposed approach.
\end{abstract}

\section{INTRODUCTION}

Simultaneous localization and mapping (SLAM) is one of the fundamental problems that need to be solved before achieving truly autonomous vehicles. For this reason, in recent years it has been the focus of a great deal of attention [1], [2]. Multiple techniques have shown promising results in a variety of different applications and scenarios: indoor, outdoor, on land and even airborne. However, the underwater environment is still one of the most challenging scenarios for SLAM because of the reduced sensorial possibilities. Acoustic devices are the most common choice, while the use of cameras and laser sensors is limited to applications where the vehicle navigates very near to the seafloor. Another important issue is the difficulty to find reliable features. There are approaches using clusters of acoustic data as features [3], [4], or merging visual and acoustic information in order to improve the reliability [5], while other strategies simply introduce artificial beacons to deal with complex environments [6]. Most of the prior work using mechanically scanned imaging sonars (MSIS) has focused on the use of point features and makes the assumption that the robot remains static or moves slowly enough to neglect the induced image distortion.

In this paper we describe an underwater SLAM system for AUVs using a MSIS that builds a feature-based map of the environment, consisting of line features corresponding to planar structures in the environment. These types of structures are present in many scenarios of interest for

This work has been funded in part by projects DPI2005-09001-C03-01 and DPI2006-13578 of the Dirección General de Investigación of Spain

D. Ribas and P. Ridao are with the Grup de Visió per Computador i Robòtica, Universitat de Girona, 17071 Girona, Spain. dribasdeia.udg.es, peredeia.udg.es

J.D. Tardós and J. Neira are with the Grupo de Robótica y Tiempo Real, Universidad de Zaragoza, 50018 Zaragoza, Spain. tardos@unizar.es, jneira@unizar.es
AUVs such as dams, ports, marinas or marine platforms. The method also removes the static assumption. This work represents a step forward in the techniques presented in [7]. First, the imaging sonar feature extraction algorithm has been completely remodelled to reduce its computational cost, to make it capable of working with the continuous flux of data from the sensor, and to improve the feature uncertainty estimation (Sections II and III). As a result, the overall SLAM algorithm have been simplified (Section IV). Finally, experimental results presenting a $600 \mathrm{~m}$ trajectory performed in a marina environment demonstrate the viability of the approach (Section V). Conclusions are presented in Section VI.

\section{WORKING WITH ACOUSTIC IMAGES}

MSISs perform scans in a 2D plane by rotating a fanshaped sonar beam through a series of small angle steps. For each emitted beam, a set of bins (distance vs. echo-amplitude values) are obtained (Fig. 1b). Accumulating this information along a complete $360^{\circ}$ sector produces an acoustic image of the surroundings (Fig. 1c). The beam typically has a large vertical beamwidth which makes possible the detection of obstacles at different heights. On the other hand, a narrow horizontal beamwidth increments the resolution of the device and improves the sharpness of the acoustic images.

\section{A. Beam segmentation}

Objects present in the environment appear as high echoamplitude returns in acoustic images. Thus, only part of the information stored in each beam is useful for feature extraction. Therefore, a segmentation process can be done in order to obtain the more significant information. This process consists in two steps. First, only those bins with an intensity value over a threshold are selected and stored. This procedure separates the acoustic imprint left by an object in the image, from the noisy background data (Fig. 2b). The resulting imprint is used to estimate the feature uncertainty as explained in Section III-D. Secondly, select among the thresholded data those bins which are local maxima and satisfy a "minimum distance between them" criterion (Fig. 2c). These local high intensity bins are the ones that most likely correspond to objects present in the scene. Thus, they are specially well suited as input to the feature extraction algorithm (Section III) while, at the same time, the computational efficiency is improved since a small number of bins are involved.

\section{B. Undistorting the acoustic images}

Commonly, MSISs have a slow scanning rate (e.g. a Tritech Miniking sonar head needs about 6 seconds to 
(a)

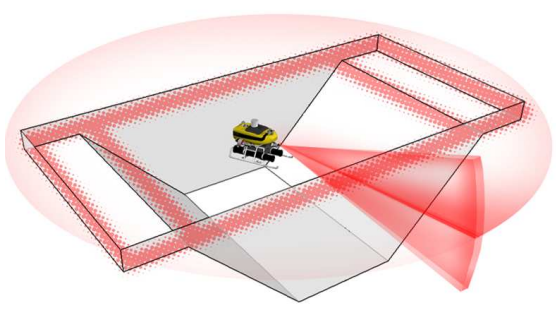

(b)

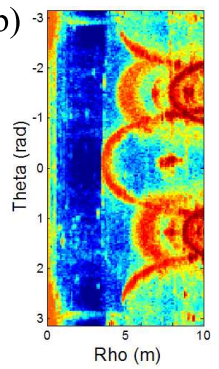

(c)

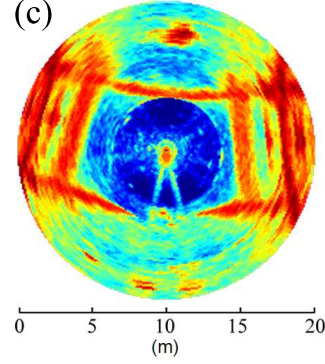

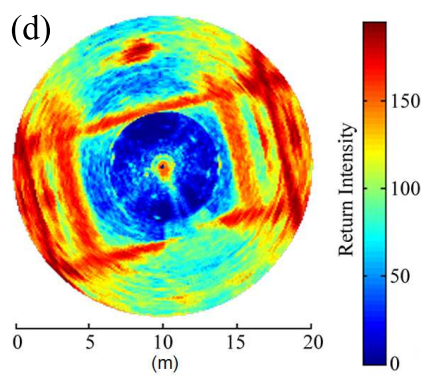

Fig. 1. (a) Schematic representation of the environment where the sonar data were gathered. (b) Raw data represented in polar coordinates. Each row corresponds to a single beam at a given orientation and each column to a bin in a particular position along the beam. (c) The same data represented in cartesian coordinates. Notice the distortion produced by the movement of the vehicle. (d) Undistorted image after integration with vehicle displacement.

(a)

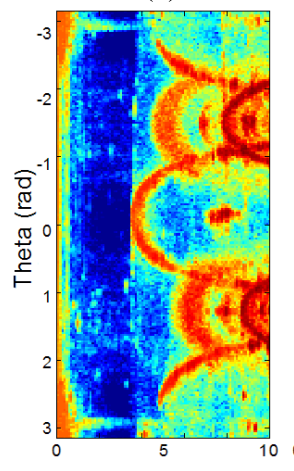

(b)

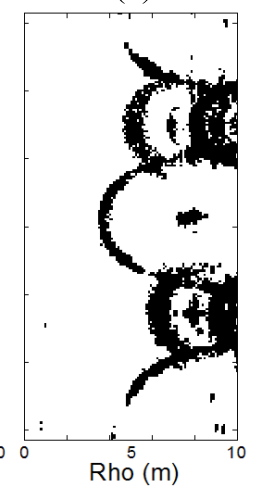

(c)

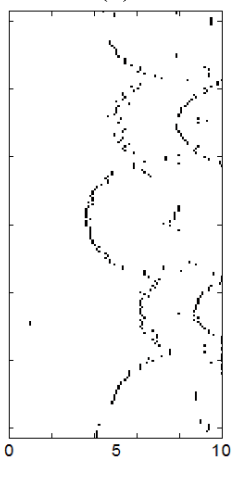

Fig. 2. (a) Raw sensor data. (b) Segmented data. (c) Local maxima bins.

complete a $\left.360^{\circ} \mathrm{scan}\right)$. For this reason, the vehicle movement along a complete scan usually induces important distortions in the acoustic image (Fig. 1c). Extracting features from this kind of images produces inaccuracies and yields poor results. Therefore, the first step of the procedure consists in merging the raw sensor data together with information regarding the vehicle movement. This information is provided by the SLAM algorithm (see Section IV) which runs simultaneously with the feature extraction algorithm. Incorporating the displacements and rotations of the sensor into the positional information of each sonar measurement results in an undistorted acoustic image (Fig. 1d).

\section{Dealing with a stream of beams}

In order to deal with the stream of measurements produced by the continuous arrival of beams, we set a data buffer to store the beams contained within the most recent $180^{\circ}$ scan sector. Whenever new beams corresponding to an unexplored zone arrive, old beams that fall outside the scan sector are discarded. The choice of a $180^{\circ}$ sector is not arbitrary since this is the maximum zone that a single line can cover within a sonar scan. Since calculations searching features are performed with every new beam (Section III), the buffer should contain the bins which are local maxima (Fig. 2c) for the line detection process, the segmented beams (Fig. 2b) for uncertainty estimation and all its associated positions in the world coordinate system to deal with the motion-induced distortions.

\section{DETECTION OF LINE FEATURES}

As said before, walls and other planar structures produce line-shaped features in acoustic images. The Hough transform [8] is a feature extraction technique which is specially well suited for this kind of situations. This algorithm accumulates the information from the sensor data into a voting table which is a parameterized representation of all the possible feature locations. Those features that receive a great number of votes are the ones with a relevant set of compatible sensor measurements and thus the ones that most likely correspond to a real object in the environment. In our application, line features are described by two parameters, $\rho^{B}$ and $\theta^{B}$ (distance and orientation with respect to a base frame B). Hence, the resulting Hough space (HS) is a twodimensional space where the voting process and the search for maxima can be done efficiently. The base reference frame B can be set arbitrarily. However, our choice for B is the position of the sensor head when the last beam arrives. So, any detected line feature will be represented directly in the sensor coordinate frame. It is worth noting that B is not a fix coordinate frame. As the parametrization in the HS is performed in polar coordinates, setting the reference in a fix position would produce loss of resolution with the increase of range. Hence, we need to resituate B near to the sensor as the vehicle moves. Unfortunately, this requires to recompute the HS with each change in the position of B. Another key issue is the quantization of the HS. In our case, we have observed that selecting the quantization equal to the angular and linear resolutions of our sensor (typically, $1.8^{\circ}$ and 0.1 m) works fine.

The feature extraction procedure works as follows: First, with each beam arrival the Hough space is initialized, and the buffered bins are referenced to B so they can be used to vote in such space. Then, the votes corresponding to each bin are assigned to the candidate lines by means of a sonar model. Finally, a search for winning candidates is performed.

\section{A. Sonar Modeling}

Each bin represents the strength of the echo intensity return in a particular place within the insonified area. Due to the uncertainty produced by the horizontal beamwidth, a measurement cannot be assigned to a single point in the 


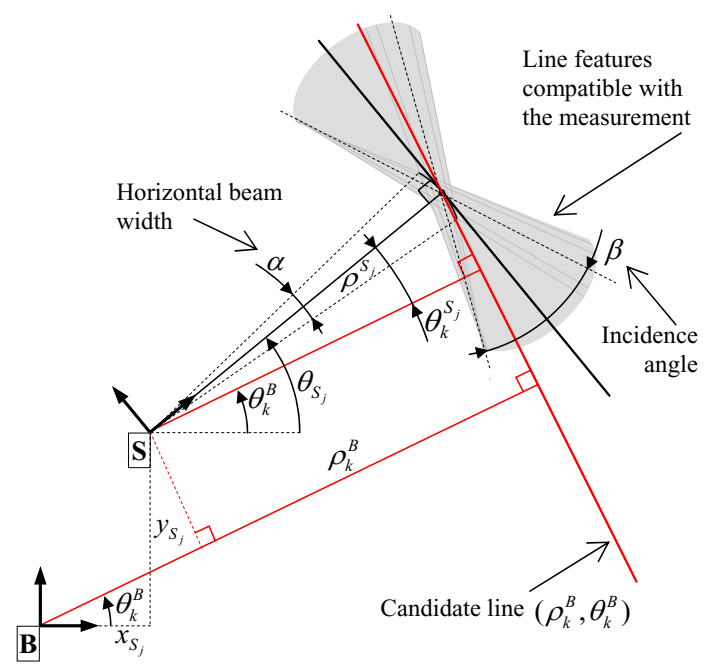

Fig. 3. Model of the sonar sensor for line features. B is the base reference frame and $\mathrm{S}$ is a reference frame attached to a beam.

space. A common approach [9],[10], is to represent a bin as an arc whose aperture represents the beamwidth uncertainty. Moreover, as a high intensity return is typically produced when the acoustic wave hits perpendicularly a surface, we can infer that all the surfaces tangent to the arc can explain the high intensity return. While this simple model is well suited for air sonar ranging systems, it is not able to explain the acoustic images gathered with a MSIS. A careful analysis of such images reveals that their object detection capability is not limited to the arc-tangent surfaces. Even those beams which meet a surface with a considerable incidence angle (for the Miniking, $\beta=60^{\circ}$ ) produce a discernible high intensity profile. For this reason, we have adopted an extended model to describe the imaging sonar. Each bin represents a zone described by an arc which corresponds to the horizontal beamwidth $\alpha$ (in our sensor, $\alpha=3^{\circ}$ ). Given a resolution and the incidence angle $\beta$, for each point belonging the arc, its tangent surface as well as the intersecting planes with an incidence angle smaller than $\pm \beta / 2$ are visible for the beam's bin (Fig. 3). Hence, the acoustic intensity represented by the bin should correspond to one of those candidate planes.

\section{B. Voting}

The next step is to determine the candidate lines that will receive the votes for each buffered bin. As previously introduced in Section III-A, the measurement is modeled as an arc in order to represent the uncertainty that appears due to the horizontal beamwith $\alpha$ (Fig. 3). Hence, $\theta_{S_{j}}$ will take values within an aperture of $\pm \alpha / 2$ around the real angle of the transducer head. Then, for each $\theta_{S_{j}}$ value, a set of $k$ candidate lines will be determined. As said before, not only the lines tangent to the arc are candidates, but also the ones inside the maximum incidence angle limits of $\pm \beta / 2$. So, for each $\theta_{S_{j}}$ value we can define $\theta_{k}^{B}$ as:

$$
\theta_{S_{j}}-\frac{\beta}{2} \leq \theta_{k}^{B} \leq \theta_{S_{j}}+\frac{\beta}{2} .
$$

Finally, the $\rho_{k}^{B}$ value that corresponds to each value of $\theta_{k}^{B}$ is calculated as:

$$
\rho_{k}^{B}=x_{S_{j}} \cos \left(\theta_{k}^{B}\right)+y_{S_{j}} \sin \left(\theta_{k}^{B}\right)+\rho^{S_{j}} \cos \left(\theta_{k}^{S_{j}}\right) .
$$

In Fig. 4 it is shown how the set of voters appear in the Hough space. Note that each selected cell of the Hough space only receives one single vote.

\section{Line extraction}

Every time a new beam arrives, a new voting space is generated to look for winning line candidates. A winning line must only be detected once it has been completely observed (i.e., further beams cannot provide more votes to the candidate). In the voting space, the zone where those winning lines can exist is completely determined by the subset of all the candidate lines contained in the most recent $180^{\circ}$ scan sector, that do not intersect with the last beam (redshaded zone in Fig. 4). Any line candidate with a sufficient number of votes that is found within this zone is declared a winner. Performing the detection in this way, we can ensure that our algorithm detects the lines as soon as they are completely visible. After a line detection, all the bins involved in the election of the winning candidate are removed from the buffer so they do not interfere with the detection of further features.

\section{Uncertainty estimation}

When a line has been detected among the candidates in the HS, the next step is to estimate its uncertainty. As the winning line has obtained a considerable number of votes, it must correspond to a high intensity zone of the buffered beams (see Fig. 2b). This zone (an arc) represents the imprint of the object (a line) in the polar representation of the acoustic image and its thickness is related to the line uncertainty. The points belonging to the arc are the polar representation of the sonar measurements corresponding to the uncertain line in Cartesian coordinates. If both, the scanner and the HS are represented in the same frame, the point with the minimum $\rho$ (the maximum of the arc) would have the same rho-theta used to represent the line in the HS. If not, a coordinate system transformation can be used to relate both of them. It is worth noting that although HS is used to represent lines, it is actually a polar representation of the space and hence points can also be represented. Lines are in fact represented using their point with smallest distance to the origin. Hence, it is possible to represent the buffered beams, for which we know its world coordinates, into the HS (referenced to B) were the detected line is represented (Fig. 5). The points belonging to the detected line describe an arc in the HS, and it is possible to compute their number of compatible measurements. Moreover, each realization of an stochastic line in Cartesian coordinates, accomplishes the following properties: (1) there exists a corresponding arc in the HS falling within the uncertain (thick) arc, (2) it has a number of compatible measurements similar to the already detected line, and (3) it is represented by a rho-theta point belonging to the neighbourhood of the already detected line. 

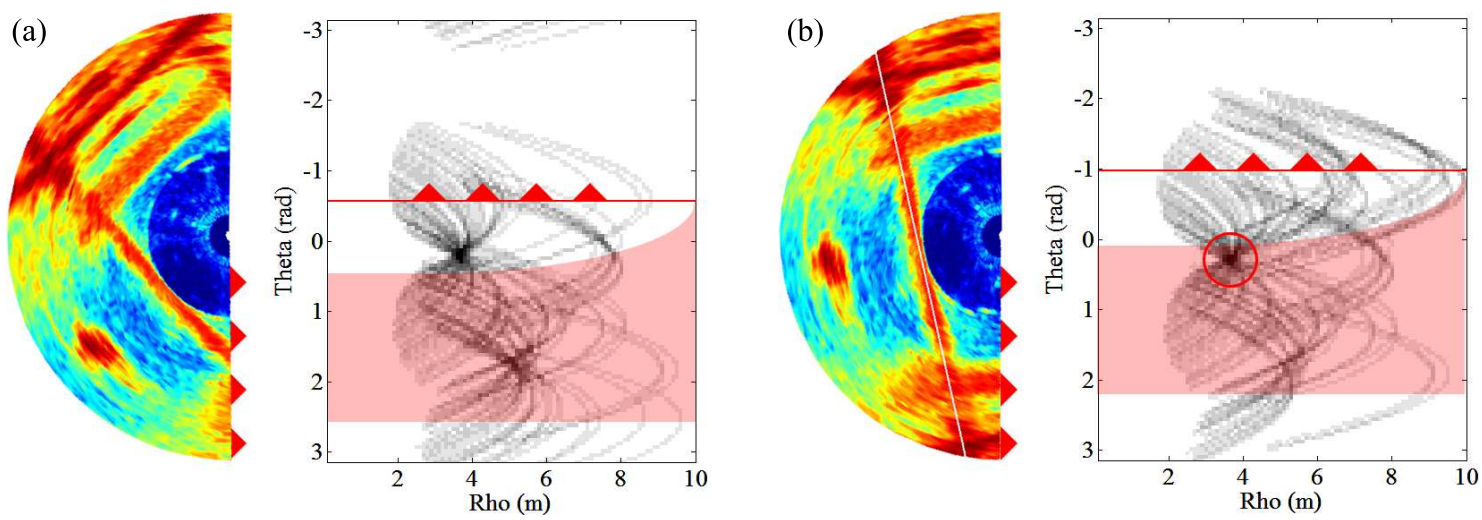

Fig. 4. Two examples of the voting process. The scan sector stored in the buffer is represented together with its corresponding generated voting space. The red triangular shapes mark the newest beam. The shaded zone represents where the candidates have received all the possible votes (a) Part of the target line is still outside the sector scan. Hence, it can receive more votes in the future. (b) The new beams can not add new votes to the target line. The line inside the shaded zone of the voting space have been fully observed and hence, it can be detected.
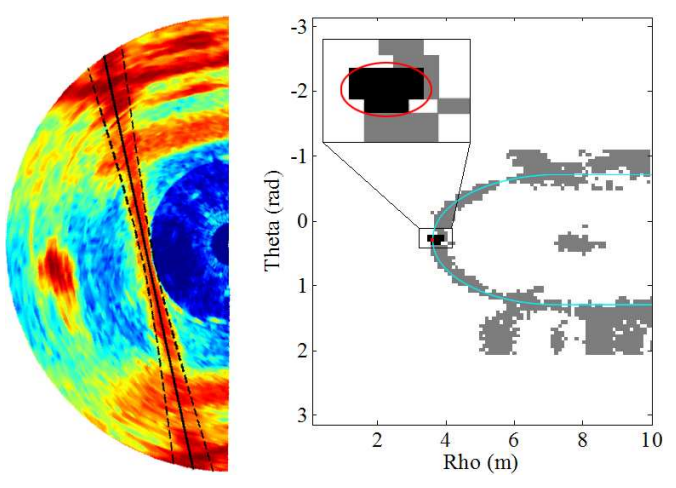

Fig. 5. Uncertainty estimation. The parameters of the winning line are shown as a small red dot in the segmented data. The line trace is in cyan. In the detail, all the neighboring compatible lines are in black. The red ellipse groups those lines and represents the feature uncertainty. The output line feature is represented in the scan sector.

Therefore, it is possible to look for the points (representing line realizations) in the neighbourhood of the detected line satisfying the 3 properties. The region described by those points has an elliptical shape in HS and it is possible to estimate its parameters (red ellipse in Fig. 5). This ellipse can then be compared with the ellipse described by a 2 dimensional multivariate normal distribution for a given confidence level. The mean of this normal distribution is taken as the output line feature, while the probability distribution itself is a representation of the feature uncertainty.

\section{SLAM ALGORITHM}

Our feature-based SLAM algorithm uses an EKF for state estimation. In this typical implementation of the stochastic map [11], the estimate of the position of both the vehicle $\mathbf{x}_{V}$ and the set of map features $\left\{\mathbf{x}_{1} \ldots \mathbf{x}_{n}\right\}$ are stored in the state vector $\hat{\mathbf{x}}$.

$$
\hat{\mathbf{x}}(k)=\left[\begin{array}{llll}
\hat{\mathbf{x}}_{V}(k) & \hat{\mathbf{x}}_{1}(k) & \ldots & \hat{\mathbf{x}}_{n}(k)
\end{array}\right]^{T} .
$$

The covariance matrix for this state is defined as:

$$
\mathbf{P}(k)=E\left([\mathbf{x}(k)-\hat{\mathbf{x}}(k)][\mathbf{x}(k)-\hat{\mathbf{x}}(k)]^{T} \mid Z(k)\right) .
$$

All the elements on the state vector are represented in the map reference frame. Although this reference frame can be defined arbitrarily, we have chosen to place its origin on the initial position of the vehicle and to orient it to the north. Hence, the compass measurements can be straight forward integrated for vehicle's heading estimation.

\section{A. Prediction}

The vehicle state $\mathbf{x}_{V}$ is represented as:

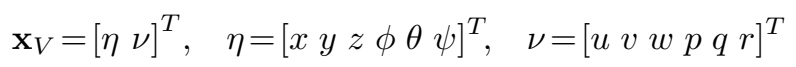

where, as defined in [12], $\eta$ is the position and attitude vector of the vehicle while $\nu$ is its linear and angular velocity vector. Note that although the vehicle's position is represented in the defined reference frame, the velocities in $\nu$ are represented in the vehicle coordinate frame. A simple 6 DOF constant velocity kinematics model is used to predict the state of the vehicle. Since AUVs are commonly operated describing rectilinear transects at constant speed during survey missions, we believe that such model is a simple but realistic way to describe the motion.

$$
\mathbf{x}_{V}(k)=f\left(\mathbf{x}_{V}(k-1), \mathbf{s}_{V}\right)
$$

where $\mathbf{s}_{V}$ is an acceleration white noise additive in the velocity with zero mean and covariance $\mathbf{Q}_{V}$. For a more detailed description of the vehicle model please refer to [7]. Features correspond to fixed objects from the environment; we assume them as stationary. Hence, the whole state can be predicted as:

$$
\hat{\mathbf{x}}(k)=\left[\begin{array}{llll}
f\left(\hat{\mathbf{x}}_{V}(k-1)\right) & \hat{\mathbf{x}}_{1}(k-1) & \ldots & \hat{\mathbf{x}}_{n}(k-1)
\end{array}\right]^{T}
$$

and its covariance matrix updated as:

$$
\mathbf{P}(k)=\left[\begin{array}{cc}
\mathbf{F}_{V} & \mathbf{0} \\
\mathbf{0} & \mathbf{I}
\end{array}\right] \mathbf{P}(k-1)\left[\begin{array}{cc}
\mathbf{F}_{V} & \mathbf{0} \\
\mathbf{0} & \mathbf{I}
\end{array}\right]^{T}+\left[\begin{array}{c}
\mathbf{G}_{V} \\
\mathbf{0}
\end{array}\right] \mathbf{Q}_{V}\left[\begin{array}{c}
\mathbf{G}_{V} \\
\mathbf{0}
\end{array}\right]^{T}
$$

where $\mathbf{F}_{V}$ and $\mathbf{G}_{V}$ are the Jacobian matrices of partial derivatives of the non-linear model function $f$ with respect to the state $\mathbf{x}_{V}$ and the noise $s_{V}$ respectively. 


\section{B. DVL update}

A SonTek Argonaut DVL unit provides bottom tracking and water velocity measurements at a frequency of $1.5 \mathrm{~Hz}$. The unit also includes a pressure sensor allowing depth estimation. The model prediction is updated by the standard Kalman filter equations with each new DVL measurement:

$$
\mathbf{z}_{D}=\left[\begin{array}{lllllll}
u_{b} & v_{b} & w_{b} & u_{w} & v_{w} & w_{w} & z_{\text {depth }}
\end{array}\right]^{T}
$$

where subindex $b$ stands for bottom tracking velocity and $w$ for through water velocity. As few or no currents are present in the application at hand, water velocity can be assumed as an approximation of the actual vehicle velocity. The measurement model is:

$$
\begin{aligned}
& \mathbf{z}_{D}=\mathbf{H}_{D} \hat{\mathbf{x}}(k)+\mathbf{s}_{D}
\end{aligned}
$$

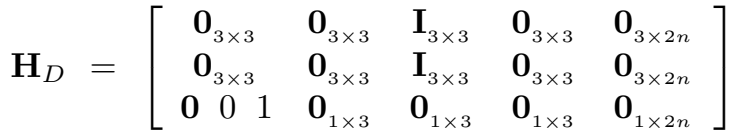

where $\mathbf{s}_{D}$ (measurement noise) is a zero-mean white noise with covariance $\mathbf{R}_{D}$. Since the DVL sensor provides a status for the bottom tracking and water velocity, depending on the quality of the measurements, different versions of the $\mathbf{H}_{D}$ matrix are used to fuse one (removing row 2), the other (removing row 1), or both readings (using the full matrix).

\section{Compass and inclinometers update}

The MTi sensor is a low cost motion reference unit (MRU) which provides attitude data at a $0.1 \mathrm{~Hz}$ rate. The current estimate can be updated with the following measurement model and the standard Kalman filter equations:

$$
\begin{aligned}
\mathbf{z}_{M}=\left[\begin{array}{cc}
\phi \theta & \psi
\end{array}\right]^{T} & , \quad \mathbf{z}_{M}=\mathbf{H}_{M} \hat{\mathbf{x}}(k)+\mathbf{s}_{M} \\
\mathbf{H}_{M} & =\left[\begin{array}{lll}
\mathbf{0}_{3 \times 3} & \mathbf{I}_{3 \times 3} & \mathbf{0}_{3 \times(6+n)}
\end{array}\right]
\end{aligned}
$$

where $\mathbf{s}_{M}$ (measurement noise) is a zero-mean white noise with covariance $\mathbf{R}_{M}$.

Compass and inclinometers are specially useful sensors for SLAM because they provide absolute measurements, unlike dead reckoning sensors normally used in SLAM such as wheel encoders, gyros or in our case the DVL. The effect of using a compass and inclinometers is threefold:

1) The error in vehicle orientation will not increase during the SLAM process.

2) Vehicle orientation introduces nonlinearity in the SLAM problem, so loss of precision because of linearization effects will also be limited.

3) Vehicle orientation errors in a certain step become position errors in future steps. Bounding the errors in orientation will also result in a reduction in the rate of increase of vehicle position errors.

Fig. 6 shows the evolution of vehicle position and orientation using the DVL velocity data together with rate of turn measurements from gyros (blue) and using absolute attitude information from the MTi (red). We can see that the error in orientation remains constant, and that there is also a reduction in the rate of increase of the error in the direction of vehicle motion.
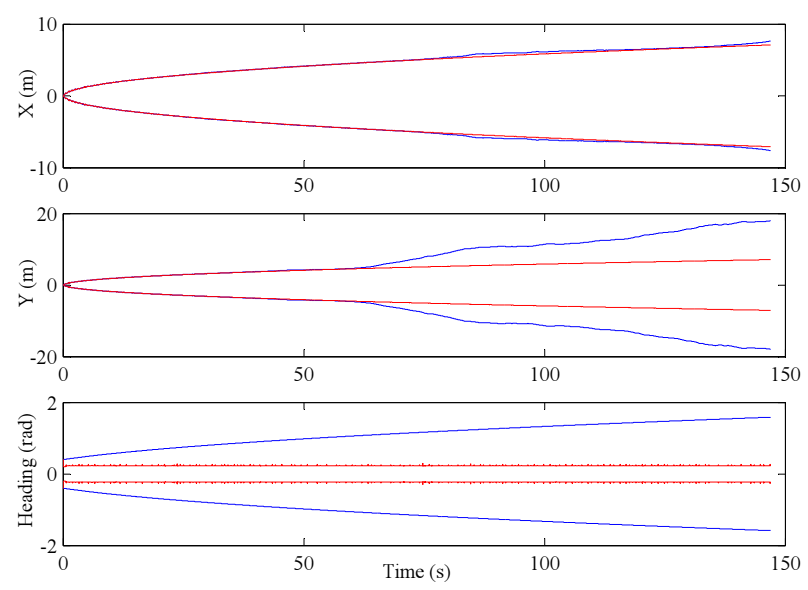

Fig. 6. Estimated position error ( $2 \sigma$ bounds). The results with a gyro sensor are represented in blue, while the ones using absolute data are in red.

\section{Imaging sonar beam arrival}

The Tritech Miniking imaging sonar produces beams at a $30 \mathrm{~Hz}$ rate. Each new beam is stored in a data buffer and fed to the feature extraction algorithm as shown in Sections II and III. Eventually, the information added by a new beam arrival is sufficient to produce a line feature detection. The rho-theta pair obtained is represented in a B frame which is placed in the sensor head. For the sake of simplicity, let us assume that the transformation between $\mathrm{B}$ and the vehicle coordinate system is known. Hence, we could represent a new feature $m$ with respect the vehicle frame $V$ as $\mathbf{x}_{m}^{V}=$ $\left[\begin{array}{ll}\rho_{m}^{V} & \theta_{m}^{V}\end{array}\right]^{T}$. However, in order to augment the state vector with the new line feature, it has to be represented in the map reference frame. This is done by compounding [11] the line feature with the current vehicle position as follows:

$$
\hat{\mathbf{x}}(k)=\left[\begin{array}{c}
\hat{\mathbf{x}}_{V}(k) \\
\vdots \\
\hat{\mathbf{x}}_{n}(k) \\
\hat{\mathbf{x}}_{m}(k)
\end{array}\right]=\left[\begin{array}{c}
\hat{\mathbf{x}}_{V}(k) \\
\vdots \\
\hat{\mathbf{x}}_{n}(k) \\
\hat{\mathbf{x}}_{V}(k) \oplus \hat{\mathbf{x}}_{m}^{V}(k)
\end{array}\right]
$$

With the new feature's uncertainty represented as $\mathbf{P}_{m}^{V}$, the whole state uncertainty is updated as:

$$
\begin{gathered}
\mathbf{P}(k)=\mathbf{F P}(k) \mathbf{F}^{T}+\mathbf{G P}_{m}^{V}(k) \mathbf{G}^{T} \\
\mathbf{F}=\left[\begin{array}{cccc}
\mathbf{I} & 0 & \ldots & 0 \\
\vdots & \vdots & \ldots & \vdots \\
0 & 0 & \ldots & \mathbf{I} \\
\mathbf{J}_{1 \oplus} & 0 & \ldots & 0
\end{array}\right] \quad \mathbf{G}=\left[\begin{array}{c}
0 \\
\vdots \\
0 \\
\mathbf{J}_{2 \oplus}
\end{array}\right]
\end{gathered}
$$

where $\mathbf{J}_{1 \oplus}$ and $\mathbf{J}_{2 \oplus}$ are the Jacobian matrices of the compounding transformation. At this point, a new line feature has been introduced into the stochastic map. The next step is to determine whether this line is a re-observation of a feature already in the map or corresponds to an object never seen before. For this purpose, every time a new line feature is detected, an individual compatibility test is performed with the other features in the map. Let us suppose that $\mathcal{H}$ is the hypothesis relating each feature in the map $\left(\hat{\mathbf{x}}_{1} \ldots \hat{\mathbf{x}}_{n}\right)$ 


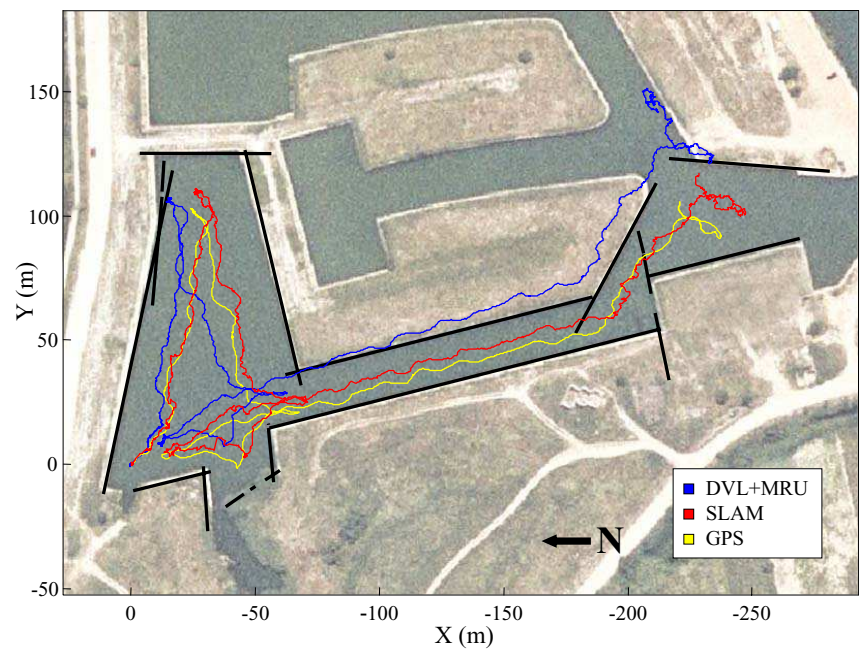

Fig. 7. The resulting map represented over a satellite image of the scenario.

with the recently observed one $\left(\hat{\mathbf{x}}_{m}\right)$. As the hypothesis relates elements belonging to the state, we can represent the condition that one line feature corresponds to another by an implicit measurement equation without noise [13]:

$$
\mathbf{z}_{\mathcal{H}}=h_{\mathcal{H}}(\hat{\mathbf{x}}(k))=0
$$

Taking into account that the Jacobian of the nonlinear function $h_{\mathcal{H}}$ is $\mathbf{H}_{\mathcal{H}}$, a compatibility test can be written as:

$$
D_{\mathcal{H}}^{2}=h_{\mathcal{H}}(\hat{\mathbf{x}}(k))^{T}\left(\mathbf{H}_{\mathcal{H}} \mathbf{P}(k) \mathbf{H}_{\mathcal{H}}^{T}\right)^{-1} h_{\mathcal{H}}(\hat{\mathbf{x}}(k))<\chi_{d, \alpha}^{2}
$$

where distance $D_{\mathcal{H}}^{2}$ is the Mahalanobis distance. The correspondence is accepted if the distance is less than $\chi_{d, \alpha}^{2}$, with $\alpha$ defined as the confidence level and $d=\operatorname{dim}\left(h_{\mathcal{H}}\right)$. The Nearest Neighbor (NN) selection criterion determines that among the features that satisfy (17), the one with the smallest Mahalanobis distance is chosen and hypothesis $\mathcal{H}$ is accepted. Having a hypothesis $\mathcal{H}$ relating compatible lines, an update of the stochastic map can be performed:

$$
\begin{aligned}
\mathbf{K}_{\mathcal{H}} & =\mathbf{P}(k) \mathbf{H}_{\mathcal{H}}^{T}\left(\mathbf{H}_{\mathcal{H}} \mathbf{P}(k) \mathbf{H}_{\mathcal{H}}^{T}\right)^{-1} \\
\hat{\mathbf{x}}(k+1) & =\hat{\mathbf{x}}(k)-\mathbf{K}_{\mathcal{H}} h_{\mathcal{H}}(\hat{\mathbf{x}}(k)) \\
\mathbf{P}(k+1) & =\left(\mathbf{I}-\mathbf{K}_{\mathcal{H}} \mathbf{H}_{\mathcal{H}}\right) \mathbf{P}(k)
\end{aligned}
$$

The newly added line feature which matched with a feature already in the map can now be eliminated from the state.

\section{EXPERIMENTAL RESULTS}

In order to test the reliability of the proposed algorithm we carried out an extensive experiment on a abandoned marina in the Costa Brava (Spain). The Ictineu AUV gathered a data set along a $600 \mathrm{~m}$ operated trajectory which included a small loop around the principal water tank and a $200 \mathrm{~m}$ straight path through an outgoing canal. The data set included measurements from the Imaging sonar, DVL and MRU sensors. For validation purposes, the vehicle was operated close to the surface attached to a GPS equipped buoy used for registering the trajectory. Fig. 7 shows the resulting map and trajectories for the experiment represented layered with a satellite image for a better interpretation of the scene. As it can be seen, the deadreckoning trajectory obtained by merging DVL and MRU data suffers from an appreciable drift (even causing it to go outside the canal). On the other hand, the SLAM-estimated trajectory is much better and corrects this defect. The set of line features from the obtained map matches almost perfectly with the real position of the marina boundaries. Comparing the result with the GPS track the similarity of the two trajectories is evident. It is worth noting that no differential signal was available to enhance the GPS during the experiment. Hence, the expected GPS accuracy was of about $15 \mathrm{~m}$.

\section{CONCLUSIONS}

An algorithm to perform SLAM in partially structured underwater environments has been presented. It takes advantage of walls and other objects typically present in some scenarios of interest to extract reliable line features from acoustic images. The main contributions of this work include a feature extraction method capable of working with the continuous stream of data from the MSIS while dealing with the distortions induced by the vehicle movement in the acoustic images, a new method for estimating their uncertainty, and the application domain. Finally, experimental results support the viability of the proposal.

\section{REFERENCES}

[1] H. Durrant-Whyte and T. Bailey, "Simultaneous localisation and mapping (SLAM): Part I, the essential algorithms," Robotics and Automation, vol. 13, no. 2, pp. 99-108, June 2006.

[2] T. Bailey and H. Durrant-Whyte, "Simultaneous localization and mapping (SLAM): Part II, state of the art," Robotics and Automation, vol. 13, no. 3, pp. 108-117, September 2006.

[3] J. Leonard, R. Carpenter, and H. Feder, "Stochastic mapping using forward look sonar," Robotica, vol. 19, p. 341, 2001.

[4] I. Tena, Y. Petillot, D. Lane, and C. Salson, "Feature extraction and data association for AUV concurrent mapping and localisation," in Proceedings ot the IEEE International Conference on Robotics and Automation, Seoul, Korea, May 2001, pp. 2785-2790.

[5] S. Williams and I. Mahon, "Simultaneous localisation and mapping on the great barrier reef," in Proceedings of the IEEE International Conference on Robotics and Automation, New Orleans, USA, AprilMay 2004.

[6] P. Newman and J. Leonard, "Pure range-only sub-sea SLAM," in Proceedings of the IEEE International Conference on Robotics and Automation, Taipei, Taiwan, September 2003, pp. 1921-1926.

[7] D. Ribas, J. Neira, P. Ridao, and J. Tardós, "SLAM using an imaging sonar for partially structured environments," in Proceedings of IEEE/RSJ International Conference on Intelligent Robots and Systems, October 2006.

[8] J. Illingworth and J. Kittler, "A survey of the hough transform," Computer Vision, Graphics, and Image Processing, Academic Press Professional, vol. 44, no. 1, pp. 87-116, 1988.

[9] J. Leonard and H. Durrant-Whyte, Directed Sonar Sensing for Mobile Robot Navigation. London: Kluwer Academic Pub., 1992.

[10] J. Tardós, J. Neira, P. Newman, and J. Leonard, "Robust mapping and localization in indoor environments using sonar data," International Journal of Robotics Research, vol. 21, no. 4, pp. 311-330, April 2002

[11] R. Smith, M. Self, and P. Cheeseman, "Estimating uncertain spatial relationships in robotics," in Autonomous robot vehicles. SpringerVerlag New York, Inc., 1990, pp. 167-193.

[12] T. Fossen, Guidance and Control of Ocean Vehicles. John Wiley \& Sons Ltd., 1994.

[13] J. Castellanos and J. Tardós, Mobile Robot Localization and Map Building: A Multisensor Fusion Approach. Kluwer Academic Publishers, Boston, 1999 